\title{
Electron-phonon renormalization in small Fermi energy systems
}

\author{
E. Cappelluti ${ }^{1,2}$, L. Pietronero ${ }^{2,3}$ \\ 1 "Enrico Fermi" Center, v. Panisperna 89a, c/o Compendio del Viminale, 00184 Roma, Italy \\ ${ }^{2}$ Dipart. di Fisica, Università di Roma "La Sapienza", \\ P.le A. Moro, 2, 00185 Roma, and INFM UdR Roma1, Italy and \\ ${ }^{3}$ CNR, Istituto di Acustica "O.M. Corbino", v. del Fosso del Cavaliere 100, 00133 Roma, Italy
}

(Dated: November 6, 2018)

\begin{abstract}
The puzzling features of recent photoemission data in cuprates have been object of several analysis in order to identity the nature of the underlying electron-boson interaction. In this paper we point out that many basilar assumptions of the conventional analysis as expected to fail in small Fermi energy systems when, as the cuprates, the Fermi energy $E_{\mathrm{F}}$ is comparable with the boson energy scale. We discuss in details the novel features appearing in the self-energy of small Fermi energy systems and the possible implications on the ARPES data in cuprates.

PACS numbers: 71.38.Cn, 63.20.Kr, 71.10.Ay
\end{abstract}

Renewed interest has recently arisen about the role of the electron-phonon (el-ph) coupling in cuprates. A powerful tool of investigation is represented by the AngleResolved Photoemission Spectroscopy (ARPES), which, in two dimensional systems as the copper oxides, is in principle able to extract the electronic self-energy directly from the momentum and.energy distribution curves 1 The aim of this analysis is to determine the microscopic origin and properties of the electron scattering. 2,3.4.5 For example, in qualitative terms, the report of a remarkable kink in the electronic dispersion has been discussed as an evidence of a retarded electron-boson interaction (most probably of phononic nature), where the energy at which the kink occurs set the energy scale of the bosonic spectrum. ${ }^{6}$ In the same framework, the ratio between the slope of the electronic dispersion at low energy (below the kink) and at high energy (above the kink) is expected to give a qualitative estimate of the strength of the electronboson coupling. 2.4 .6

Some puzzling features question however this conventional el-ph picture.On one hand, the low energy electronic dispersion shows a quite weak dependence on the hole doping in contrast with a significant dependence of the apparent el-ph coupling constant ${ }^{7}$ More interesting, the high energy electronic dispersion results to be strongly dependent on the hole doping $\frac{7}{7}$ This is particular astonishing because the high energy part of the electronic dispersion is expected to represent the bare electronic structure and it should not be dependent on any electron-phonon properties. ${ }^{8.9}$ In this situation the attempt to fit this experimental scenario within a conventional el-ph framework by means of inversion techniques of the raw data would lead to unrealistic assumptions for the hypothetical underlying electron-boson spectrum 10.11

The reliability of a conventional analysis concerning the electron-phonon properties in cuprates is questioned also on the theoretical ground. Due to the high degree of the electronic correlation, the charge carriers in cuprates are characterized by a weakly dispersive effective band, with a Fermi energy $E_{\mathrm{F}} \simeq 0.3-0.4 \mathrm{eV} .12$ Similar es- timates of $E_{\mathrm{F}}$ are obtained by penetration depth measurements as reported in Uemura's plot 13 This value should be compared with the highest phonon frequencies in cuprates $\omega_{\mathrm{ph}}^{\max } \simeq 80-100 \mathrm{meV}$, defining an adiabatic ratio $\omega_{\mathrm{ph}}^{\max } / E_{\mathrm{F}} \sim 0.2-0.3$. In this situation, the adiabatic assumption $\left(\omega_{\mathrm{ph}} \ll E_{\mathrm{F}}\right)$, on which the conventional elph analysis relies, breaks down and novel nonadiabatic interferences between the electronic and lattice degrees of freedom are expected to affect the normal and superconducting state phenomenology ${ }^{14}$ Among other features, the appearance of nonadiabatic effects has shown to account in a natural way for the presence of an finite isotope effect on the effective electron mass and for the possibility of high- $T_{c}$ superconductivity within an el-ph scenario 15,16

Aim of the present paper is to investigate in some detail how the presence of a small Fermi energy, of the same order of the phonon frequencies, affects the electron-phonon phenomenology. As a first step in this direction, we do not consider here the onset of nonadiabatic vertex diagrams which arise in the nonadiabatic regime, ${ }^{14.15}$ but we retain only the nonadiabatic effects related to the finite electronic bandwidth. Note however that, while vertex diagrams play a fundamental role in determining an effective enhancement of the superconducting pairing 15 finite bandwidth effects alone have been shown to account in a qualitative way for the anomalous el-ph effects in different normal state properties (effective mass $m^{*}$, Pauli spin susceptibility, ldots) ${ }^{16,17}$. We anticipate here, as our main results, that a conventional (adiabatic) el-ph analysis needs to be deeply revised in small Fermi energy systems. In particular, we show that the following fundamental el-ph properties ${ }^{8,9}$ are no longer valid when $E_{\mathrm{F}} \sim \omega_{\mathrm{ph}}:$

(i) the el-ph self-energy $\Sigma(\omega)$ does not renormalize the electronic dispersion for $\omega$ much larger than the phonon energy scale $\omega_{\mathrm{ph}}$;

(ii) impurity scattering affects only the imaginary part of the self-energy but not the real part, and hence not the electronic dispersion; 
(iii) different channels of electron scattering (phonons, impurities, ... ) just sum in the self-energy.

Working tools of our analysis will be the MarsiglioSchossmann-Carbotte (MSC) equations 18 properly generalized in the case of finite bandwidth. The formal derivation of the MSC iterative procedure in the case of finite Fermi energy systems follows quite closely the steps outlined in Refs. 1819. Here we report only the final equations. The procedure can be derived in full generality for any shape of the density of states (DOS) and any electron filling. For sake of simplicity, and in order to disentangle finite bandwidth effects from the breaking of particle-hole symmetry, we consider a simple system at half-filling with a constant DOS: $N(\epsilon)=N(0)$ for $|\epsilon| \leq E_{\mathrm{F}}$, where the half-bandwidth represents also the Fermi energy In the presence of both el-ph interaction and impurity scattering, MSC equations read thus:

$$
\begin{aligned}
\Sigma\left(i \omega_{n}\right)= & -2 i T \sum_{m} \lambda\left(i \omega_{n}-i \omega_{m}\right) \eta\left(\omega_{m}\right)-2 i \gamma \eta\left(\omega_{n}\right)(1) \\
\Sigma^{\prime}(\omega)= & 2 T \sum_{m} \lambda^{\prime}\left(\omega, \omega_{m}\right) \eta\left(\omega_{m}\right)-\int_{-\infty}^{\infty} d \Omega \alpha^{2} F(\Omega) \\
& \times[N(\Omega)+f(\Omega-\omega)] \eta^{\prime}(\omega-\Omega)-\gamma \eta^{\prime}(\omega),(2) \\
\Sigma^{\prime \prime}(\omega)= & -\int_{-\infty}^{\infty} d \Omega \alpha^{2} F(\Omega)[N(\Omega)+f(\Omega-\omega)] \\
& \times \eta^{\prime \prime}(\omega-\Omega)-\gamma \eta^{\prime \prime}(\omega),
\end{aligned}
$$

where $N(x)$ and $f(x)$ are respectively the Bose and Fermi distribution functions, $\alpha^{2} F(\Omega)$ is the el-ph Eliashberg function, $\gamma$ the impurity scattering rate. Moreover $\lambda(z)=\int_{-\infty}^{\infty} d \Omega \alpha^{2} F(\Omega) /[\Omega-z]$ ( $z$ complex number $)$, $\lambda^{\prime}\left(\omega, \omega_{m}\right)=\operatorname{Im} \lambda\left(\omega-i \omega_{m}\right)$, and

$\eta\left(\omega_{m}\right)=\arctan \left[\frac{E_{\mathrm{F}}}{\omega_{m} Z\left(\omega_{m}\right)}\right]$,

$\eta^{\prime}(\omega)=\frac{1}{2} \ln \left[\frac{\left[E_{\mathrm{F}}-\omega Z^{\prime}(\omega)\right]^{2}+\left[\omega Z^{\prime \prime}(\omega)\right]^{2}}{\left[E_{\mathrm{F}}+\omega Z^{\prime}(\omega)\right]^{2}+\left[\omega Z^{\prime \prime}(\omega)\right]^{2}}\right]$,

$\eta^{\prime \prime}(\omega)=\arctan \left[\frac{E_{\mathrm{F}}-\omega Z^{\prime}(\omega)}{\omega Z^{\prime \prime}(\omega)}\right]+\arctan \left[\frac{E_{\mathrm{F}}+\omega Z^{\prime}(\omega)}{\omega Z^{\prime \prime}(\omega)}\right]$,

where $Z(z)=1-\Sigma(z) / z$. As usual, the self-energy on the real axis is obtained by using the Matsubara solution [Eq. (11)] as input in Eqs. (2)-(3). Note that, in the normal state, this is necessary only as long as the Fermi energy $E_{\mathrm{F}}$ is finite. Note in addition that also the self-consistency of Eqs. (2) - (3) is intrinsically related to the finiteness of $E_{\mathrm{F}}$, so that the factors $\eta^{\prime}(\omega), \eta^{\prime \prime}(\omega)$ are functions of the self-energy itself through the $Z(\omega)$ function. As we are going to see, it is just the non-linear self-consistent relation of Eqs. (2)-(3) which is responsible for the violation of the conditions $(i)-(i i i)$.

In order to underline the small Fermi energy effects on the el-ph spectral properties, let us consider for the moment a simple Einstein phonon mode with phonon frequency $\omega_{0}$ in the absence of impurities. In Fig. 17 we show the real and imaginary part of the self-energy for
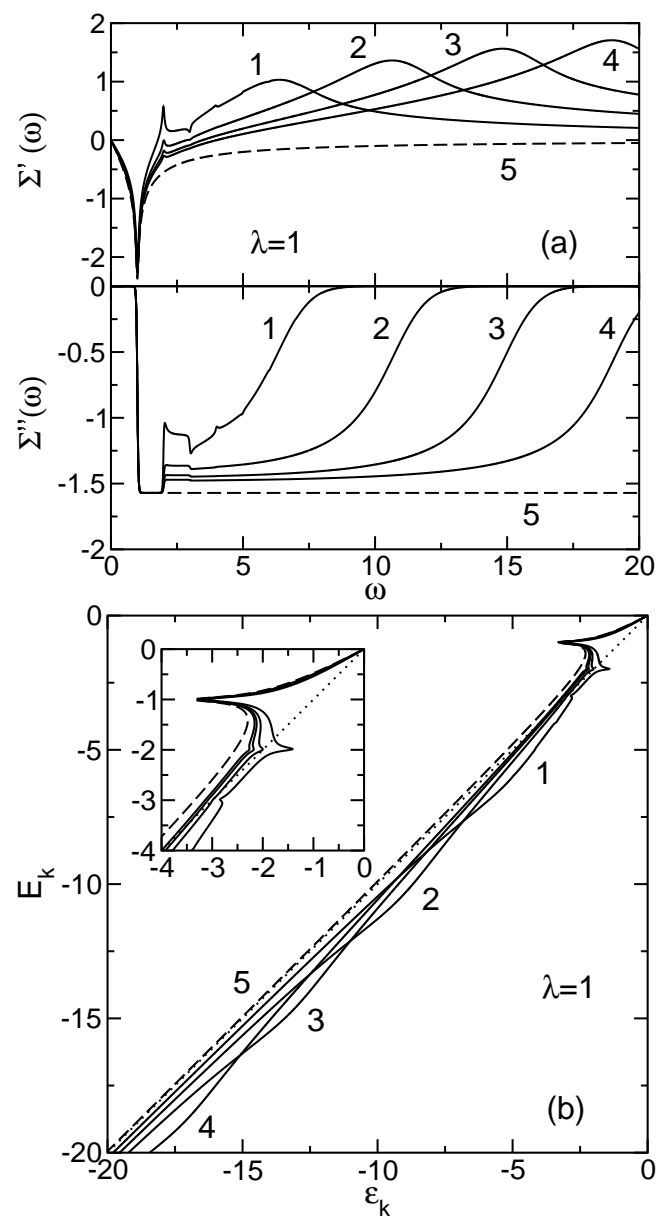

FIG. 1: Panel (a): Real and imaginary part of the self-energy for a Einstein phonon mode with $\lambda=1$ and different Fermi energy. Solid lines from 1-4: $E_{\mathrm{F}}=4,8,12,16 \omega_{0}$; Dashed line 5: $E_{\mathrm{F}}=\infty$. All quantities are in units of $\omega_{0}$. Panel (b): corresponding renormalized electron dispersion $E_{\mathbf{k}}$ as function of the bare one $\epsilon_{\mathbf{k}}$ (dotted line). In the inset a zoom of the low energy part.

, an Einstein spectrum with $\lambda=\int_{-\infty}^{\infty} d \Omega \alpha^{2} F(\Omega) / \Omega=1$ and different Fermi energies. The dashed lines represent the case of the conventional $E_{\mathrm{F}}=\infty$ el-ph: the low energy part of $\Sigma^{\prime}(\omega)$ is just $\Sigma^{\prime}(\omega) \sim \lambda \omega$, which gives the well-known the renormalization of the electronic dispersion $E_{\mathbf{k}}=\epsilon_{\mathbf{k}} /(1+\lambda)$ close to the Fermi level $\stackrel{8.9}{\text { Note }}$ that the real part of the self-energy is always negative implying that the effective electronic band $E_{\mathbf{k}}$ is always less steep than the bare one $\epsilon_{\mathbf{k}}: E_{\mathbf{k}} \leq \epsilon_{\mathbf{k}}$ for any energy. Note also that the magnitude of $\Sigma^{\prime \prime}(\omega)$ is a monotonously increasing function with $\omega$ and saturates for $\omega \geq \omega_{\mathrm{ph}}^{\max }$.

The presence of a Fermi energy of the same order of the phonon frequencies gives rise to a number of anomalous features. The most commonly known is the reduction of the low energy el-ph renormalization $E_{\mathbf{k}}=\epsilon_{\mathbf{k}} /\left(1+\lambda_{\text {eff }}\right)$, where $\lambda_{\text {eff }}=-\lim _{\omega \rightarrow 0} \Sigma^{\prime}(\omega) / \omega$ is shown, already by a simple Matsubara analysis, to be less than $\lambda$ due to finite bandwidth effects. More interesting, we note two 
qualitatively new features which appear for $\omega_{0} / E_{\mathrm{F}} \neq 0$. The first one is that $\Sigma^{\prime \prime}(\omega)$ is no longer a monotonous function of $\omega$, but when $\omega$ becomes roughly $\omega \gtrsim E_{\mathrm{F}}$ the imaginary part of the self-energy starts to decrease and it goes quite rapidly to zero. This is easily understandable in small Fermi energy systems if one considers that for $\omega \gg E_{\mathrm{F}}$ there are no electronic states into which an electron with energy $\omega$ could decay within an energy window $\sim \omega_{0}$. Another interesting feature is the large positive hump of the real part of the self-energy occurs by the Kramers-Kronig relations in correspondence of the drop of the imaginary part and it scales with $E_{\mathrm{F}}$. In particular we note that, in contrast with the case $E_{\mathrm{F}}=\infty$, for finite $E_{\mathrm{F}}$ the real part of the self-energy $\Sigma^{\prime}(\omega)$ becomes positive in a large range of energy for $\omega \gtrsim 2 \omega_{0}$. The positiveness of $\Sigma^{\prime}(\omega)$ has important consequences on the renormalized electronic dispersion obtained by $E_{\mathbf{k}}-\epsilon_{\mathbf{k}}-\Sigma^{\prime}\left(E_{\mathbf{k}}\right)=0$ which corresponds in ARPES measurements to the dispersion inferred by the momentum distribution curves (MDC). As shown in Fig. Пo the positive part of $\Sigma^{\prime}(\omega)$ implies an "anti-rinormalization" of the electron band, namely $E_{\mathbf{k}}>\epsilon_{\mathbf{k}}$. This new feature extends up to an energy scale which does not depend on $\omega_{0}$ but only on $E_{\mathrm{F}}$, while its magnitude depends on el-ph parameters as $\lambda$ or $E_{\mathrm{F}}$ itself. In such a situation the high energy part $E_{\mathbf{k}}>\omega_{\mathrm{ph}}$ of the experimental electronic dispersion ${ }^{6.7}$ does not represent anymore the bare band $\epsilon_{\mathbf{k}}$ but it expected to show a steeper behavior than $\epsilon_{\mathbf{k}}$. As a last observation, note the kinks/jumps in the real and imaginary parts of the self-energy occurring at the multiples of $\omega_{0}$. These anomalies were predicted already in Ref. 9

As we have just shown, in small Fermi energy system particular care is needed in order to disentangle el-ph properties from the knowledge of the renormalized electronic dispersion as one could get from ARPES. This issue is hardened by the fact that other actors can play an important role on the renormalization of the electron band. Most important is the presence of disorder and impurities. In conventional metals where $E_{\mathrm{F}}$ is much larger than any other energy scale impurity scattering can be considered in good approximation as static: it provides a finite quasi-particle lifetime in the imaginary part of the self-energy, but it does not affect the real part of $\Sigma$, and consequently the electronic dispersion which can be thought to be determined only by the retarded (boson mediated) scattering. Things are different in small Fermi energy systems.

In Fig. 2 2 we plot the real and imaginary part of the self-energy for an el-ph Einstein model with $E_{\mathrm{F}}=4 \omega_{0}$ and $\lambda=1$ in the presence of impurity scattering. In the contrast with the $E_{\mathrm{F}}=\infty$ case we see that the impurity scattering has important effects on the real part of $\Sigma$. On one hand it smooths the el-ph resonance at $\omega=\omega_{0}$ as well as the additional ones at $\omega=n \omega_{0}$. On the other hand it significantly enhances the positive part of $\Sigma^{\prime}(\omega)$ for $\omega \gg \omega_{0}$. The difficulty to extract in a correct way information about the el-ph spectrum $\alpha^{2} F(\Omega)$ from the (ARPES-like) $E_{\mathbf{k}}$ vs. $\epsilon_{\mathbf{k}}$ is thus even higher than in
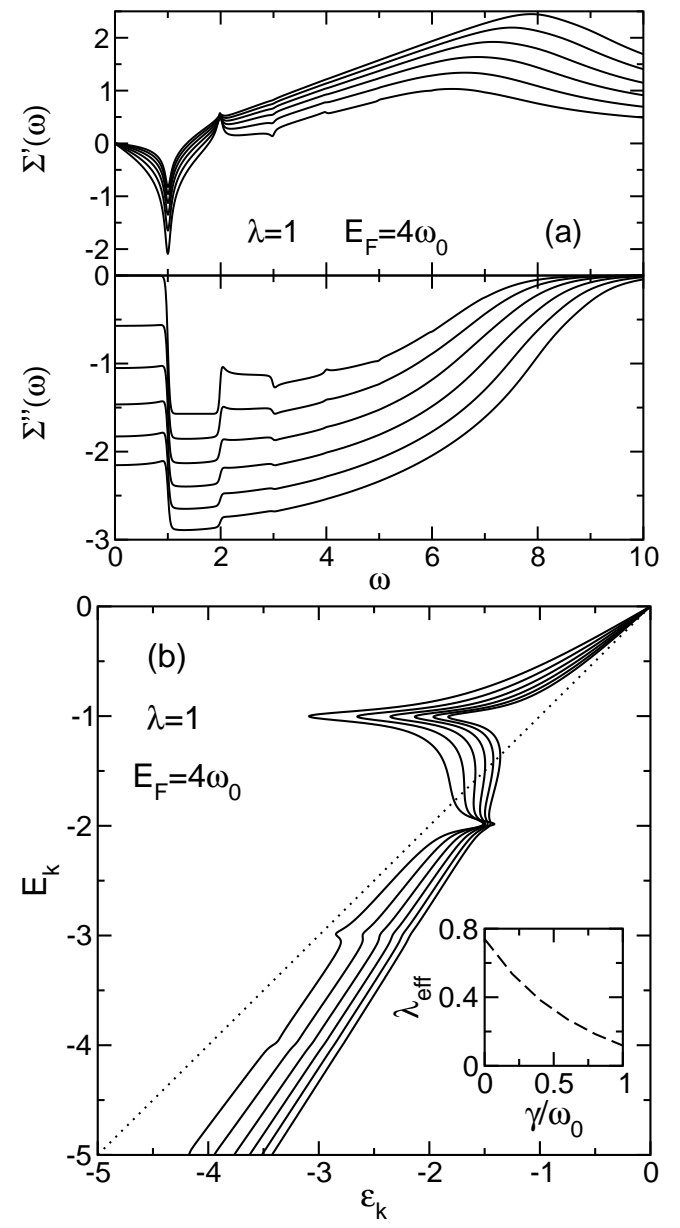

FIG. 2: Panel (a): Real and imaginary part of $\Sigma$ for a Einstein phonon mode with $\lambda=1$ and $E_{\mathrm{F}}=4 \omega_{0}$ in the presence of impurity scattering. Solid lines corresponds to (upper panel: from bottom to the top; lower panel: from top to the bottom): $\gamma / \omega_{0}=0,0.2,0.4, \ldots, 1.0$ where $\gamma$ is the impurity scattering rate. Energy quantities are expressed in units of $\omega_{0}$. Panel (b): renormalized electron dispersion corresponding (from left to the right) to panel (a). Inset: dependence of the effective parameter $\lambda_{\text {eff }}$ on the impurity scattering rate.

the absence of impurities, as we show in Fig. 20. In particular one should take into account that neither the magnitude of the kink at $\omega=\omega_{0}$ neither its broadness are directly related anymore to the properties of the el-ph spectrum $\alpha^{2} F(\Omega)$ like its total strength $\sim \lambda$ and its frequency shape. To be more specific, even the low and high energy parts of $E_{\mathbf{k}}$ are affected in a remarkable way by the impurity scattering. In the inset we plot the dependence of the "effective" $\lambda_{\text {eff }}=-\lim _{\omega \rightarrow 0} \Sigma^{\prime}(\omega) / \omega$ as function of the impurity scattering rate. We note thus that if the slope of the low energy part were used to extract the el-ph coupling, we would get a strong underestimation of $\lambda$ In addition, the discrepancy between $E_{\mathbf{k}}$ and the bare dispersion $\epsilon_{\mathbf{k}}$ at high energy is even larger in the presence of impurities so that a steeper bare electron dispersion than the real one would be predicted if the high energy 


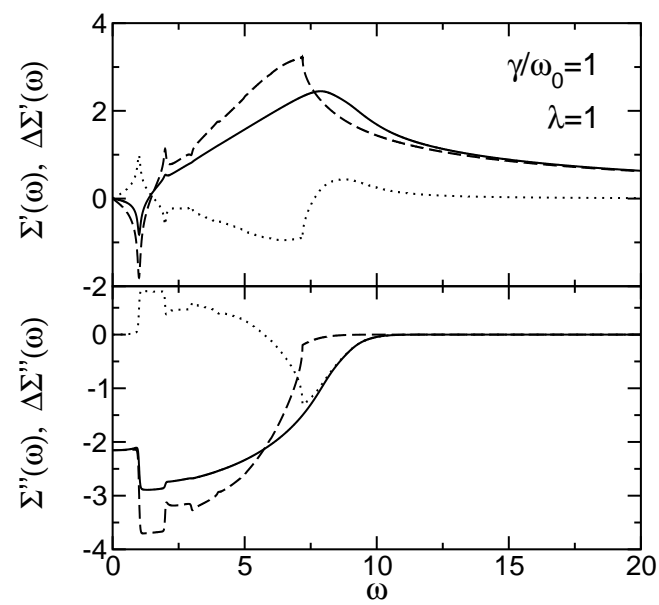

FIG. 3: Real and imaginary part of the self-energy $\Sigma_{\text {el-ph+imp }}$ an el-ph + impurities system with $\lambda=1, \gamma=\omega_{0}$ and $E_{\mathrm{F}}=4 \omega_{0}$ (solid line), as compared with the self-energy obtained by summing independent contributions $\Sigma_{\mathrm{el}-\mathrm{ph}} * \Sigma_{\mathrm{imp}}$ (dashed line). The dotted line represents the difference $\Delta \Sigma=\Sigma_{\mathrm{el}-\mathrm{ph}+\mathrm{imp}}-\Sigma_{\mathrm{el}-\mathrm{ph}}-\Sigma_{\mathrm{imp}}$ which is related to the non-linear feedback arising in small Fermi energy systems.

part of $E_{\mathbf{k}}$ to estimate it. For instance, for $E_{\mathrm{F}}=4 \omega_{0}$, $\lambda=1$ and $\gamma / \omega_{0}=1$ we would get $E_{\mathbf{k}}^{\text {high }} \sim 1.6 \epsilon_{\mathbf{k}}$.

As a last point in this paper, we would like to stress the non-linear and self-consistent nature of the normalstate MSC equations (2)-(3) in the presence of a finite Fermi energy. In fact, when $E_{\mathrm{F}} \rightarrow \infty, \eta^{\prime}(\omega)=0$, $\eta^{\prime \prime}(\omega)=\pi \operatorname{sgn}(\omega)$, the right-hand side of Eqs. (2)(3) do not depend anymore on the function $Z(\omega)$, and the different channels of interaction (el-ph, impurities, spin fluctuations etc...) just sum up linearly, so that $\Sigma(\omega)=\Sigma_{\text {el-ph }}(\omega)+\Sigma_{\text {imp }}(\omega)+\Sigma_{\text {spin }}(\omega)+\ldots$, where each term can be evaluated in the absence of the other interaction channels. Due to the self-consistent feedback of $\eta^{\prime}$ and $\eta^{\prime \prime}$, this is no more true in small Fermi energy systems, and a simplistic analysis where the self-energy was fitted by a sum of independent different contributions is expected to fail. To illustrate explicitly this point we show in Fig. 3 the total self-energy $\Sigma_{\text {el-ph+imp for }}$ an el-ph + impurities system with $\lambda=1, \gamma=\omega_{0}$ and $E_{\mathrm{F}}=4 \omega_{0}$, in comparison with the self-energy obtained as sum of independent contributions $\Sigma_{\text {el-ph }}+\Sigma_{\text {imp }} \equiv$ $\Sigma\left(\lambda=1, \gamma / \omega_{0}=0\right)+\Sigma\left(\lambda=0, \gamma / \omega_{0}=1\right)$. As we see, the discrepancies between the fully self-consistent selfenergy and its approximation as sum of two independent contributions can be quite important in the whole range of energy $\omega$. In particular we note in the imaginary part that the jump at $\omega=\omega_{0}$ due to the onset of el-ph decay processes can be much smaller than what expected by the simple sum of independent el-ph and impurity contributions. This observation thus questions the possibility to estimate the el-ph coupling by difference between $\left.\Sigma_{\mathrm{el}-\mathrm{ph}}(\omega)>\omega_{\mathrm{ph}}^{\max }\right)$ and $\Sigma_{\mathrm{el}-\mathrm{ph}}(\omega=0)$ if the other contributions could be disentangled, in the contrast with the $E_{\mathrm{F}}=\infty$ case where this difference is simply related to $\lambda\langle\omega\rangle$. We also note however that, due to the vanishing of the el-ph processes, the limit $\omega \rightarrow 0$ of the self-energy is not affected by the interplay between the different channels of interaction, but it is mainly determined by the only impurity scattering. It can be still thus used in a safe way to estimate $\gamma$.

In conclusion, motivated by the puzzling features of recent ARPES measurements in the cuprates, in this paper we have revised the el-ph properties in small Fermi energy systems with $E_{\mathrm{F}}$ of the same order of the phonon frequencies, and the interplay with other kinds of scattering, as impurity-like. We find that many basilar assumptions which are valid in conventional metals where $E_{\mathrm{F}} \gg \omega_{\mathrm{ph}}, \gamma$ need to be strongly reconsidered. In more details we have shown that in small Fermi energy:

- a positive part of the self-energy is predicted for $\omega \gtrsim \omega_{\text {ph}}$. This implies an "anti-rinormalization" of the electronic band for $\omega_{\mathrm{ph}} \lesssim \omega \lesssim E_{\mathrm{F}}$. ARPES data are expected to measure thus in this regime an electronic dispersion steeper than the bare one;

- impurity scattering can significantly affect the real part of the self-energy and hence the electronic dispersion. In particular the effective renormalization of low energy part of the electronic dispersion is reduced by the impurity scattering which can also "wash out" the el-ph kink at $\omega \simeq \omega_{\text {ph}}$;

- different scattering channels are not additive as in the case of conventional systems. Fitting data by using a sum of independent self-energy contribution is expected to fail.

Taking into account all these effects in a compelling way would make more complex but also more interesting the analysis of ARPES data in cuprates and it could contribute to understand many puzzling features which are still open issues in these compounds. We would like to finally stress that the present analysis is not restricted to the el-ph case, but it applies equally well for any retarded interaction independently of the specific origin of the bosonic mediator.

This work was partially supported by PRA-UMBRA project of INFM and FIRB RBAU017S8R of MIUR.
1 Z.-X- Shen and Dessau, Phys. Rep. 253, 1 (1995).

2 M. Hengsberger et al., Phys. Rev. Lett. 83, 582 (1999).

${ }^{3}$ M.R. Norman et al., Phys. Rev. B 60, 7585 (1999).
${ }^{4}$ S. LaShell, E. Jensen, and T. Balasubramanian, Phys. Rev. B 61, 2371 (2000).

5 J. Shi et al., cond-mat/0308055 (2003). 
6 A. Lanzara et al., Nature 412, 510 (2001).

7 X.J. Zhou et al., Nature 423, 398 (2003).

8 G. Grimvall, The Electron-Phonon Interaction in Metals (North-Holland, Amsterdam, 1981).

9 S. Engelsberg and J.R. Schrieffer, Phys. Rev. 131, 993 (1963).

10 S. Verga, A. Knigavko, and F. Marsiglio, Phys. Rev. B 67, 054503 (2003).

11 E. Schachinger, J.J. Tu, and J.P: Carbotte, Phys. Rev. B 67, 214508 (2003).

12 E. Dagotto, Rev. Mod. Phys. 66, 763 (1994).

13 Y.J. Uemura et al., Nature 352, 605 (1991).
14 L. Pietronero et al., Phys. Rev. B 52, 10516 (1995); ibid. 52, 10530 (1995).

15 C. Grimaldi et al., Phys. Rev. Lett. 75, 1158 (1995).

16 C. Grimaldi, E. Cappelluti, and L. Pietronero, Europhys. Lett. 42, 667 (1998).

17 E. Cappelluti, C. Grimaldi and L. Pietronero, Phys. Rev. B 64, 125104 (2001).

18 F. Marsiglio, M. Schossmann, and J. P. Carbotte, Phys. Rev. B 37, 4965 (1988).

19 J.K. Freericks et al., Phys. Rev. B 55, 11651 (1997). 